\title{
Surgical management of the neurogenic bladder after spinal cord injury
}

\author{
Jean-Jacques Wyndaele ${ }^{1}$ (D) $\cdot$ Brian Birch $^{2} \cdot$ Albert Borau $^{3} \cdot$ Frank Burks $^{4} \cdot$ David Castro-Diaz $^{5} \cdot$ \\ Emmanuel Chartier-Kastler ${ }^{6} \cdot$ Marcus Drake $^{7}$. Osamu Ishizuka ${ }^{8} \cdot$ Tomonori Minigawa $^{8} \cdot$ Eloy Opisso $^{3}$. \\ Kenneth Peters ${ }^{4} \cdot$ Barbara Padilla-Fernández ${ }^{5} \cdot$ Christine Reus $^{6,7,8,9,10,11} \cdot$ Noritoshi Sekido $^{9}$
}

Received: 29 December 2017 / Accepted: 5 April 2018 / Published online: 21 April 2018

c) Springer-Verlag GmbH Germany, part of Springer Nature 2018

\begin{abstract}
Purpose This work represents the efforts of the SIU-ICUD workgroup on this topic and comprehensive literature search of English language manuscripts regarding urologic surgery in spinal cord injury using key words of urologic surgery and spinal cord injury. Articles were compiled, and recommendations in the chapter are based on group discussion and intensive communication. The purpose is to review what has been published during the last decades on urological surgery for neurogenic bladder after spinal cord injury.

Methods Surgical techniques applied in spinal cord injured patients for neurogenic bladder dysfunction have been reviewed and the published material evaluated.

Results There are several techniques that can be used to treat neurogenic dysfunctions and symptoms in refractory cases where conservative treatment failed. The number of publications is small as are the number of patients with spinal cord injury in which they have been performed. The choice of techniques proposed to the patients depends on the exact functional pathology in bladder, bladder neck and urethral sphincter. The final informed choice will be made by the patient.
\end{abstract}

Conclusion There are surgical urological techniques available to treat neurologic dysfunctions in spinal cord injured patients.

Keywords Surgery $\cdot$ Lower urinary tract $\cdot$ Spinal cord injury

Jean-Jacques Wyndaele

Wyndaelejj@skynet.be

1 University Antwerp, Antwerp, Belgium

2 Department of Urology, University Hospital Southampton and University of Southampton School of Medicine, Southampton, USA

3 Fundació Institut Guttmann, Universitat Autónoma de Barcelona, Barcelona, Spain

4 Department of Urology, Beaumont Health, Oakland University William Beaumont School of Medicine, Royal Oak, USA

5 Urology, Universidad de La Laguna, Complejo Hospitalario Universitario de Canaris, San Cristóbal de La Laguna, Spain

6 Department of Urology, Pitié-Salpêtrière Academic Hospital, Assistance Publique-Hôpitaux de Paris, Pierre and Marie Curie Medical School, Sorbonne Universités, Paris, France
7 School of Clinical Sciences, University of Bristol, Bristol, UK

8 Department of Urology, Shinshu University School of Medicine, Nagano, Japan

9 Department of Urology, School of Medicine, Faculty of Medicine, Toho University, Toho University Ohashi Medical Center, Tokyo, Japan

10 Department of Molecular Medicine and Surgery, Section of Urology, Karolinska Institutet, Stockholm, Sweden

11 Department of Urology, Karolinska University Hospital, Stockholm, Sweden 


\section{Introduction}

Neurogenic bladder dysfunction (NBD) is highly prevalent in individuals with spinal cord injury (SCI). Although treatment is usually non-surgical, such treatment modality does not have a good outcome in some patients: e.g., urinary incontinence and elevated bladder pressures can necessitate a more aggressive treatment. Urinary incontinence can occur as the result of sphincter incompetence, involuntary detrusor contraction, or poor compliance. Elevated bladder pressure can be caused by loss of compliance, neurogenic detrusor overactivity (NDO), and/or detrusor sphincter dyssynergia (DSD). Dysfunctions are not the same in all SCI individuals with a specific level and grade of the spinal cord lesion. This makes a division in techniques based mainly on symptoms or on spinal cord level not clinically useful.

It is mandatory to list the urologic symptoms and fully evaluate the function of the bladder, bladder neck, and urethral sphincter before a decision is made about which surgery should be proposed. Surgery does not have to be a stand-alone option; it can be paired with drug treatment and catheterization as necessary. The level and completeness of the lesion, a urological history, and informed consent of the patient are all important.

Different surgical techniques have been developed for correction of neurogenic detrusor, bladder neck, and sphincter dysfunction refractory to a conservative approach. The evidence regarding the procedure and recommendations for or against its use in SCI patients is presented. Less invasive techniques as injection of botulinum toxin, are not included in the list.

\section{Bladder neck procedures}

The purpose of these procedures is either to tighten the bladder neck to aid in continence, or to open the bladder neck to cause incontinence and protect the kidneys from elevated bladder pressure.

\section{Injection of bulking agents in the bladder neck}

Dextranomer/hyaluronic acid copolymer, polytetrafluoroethylene, and collagen have all been used for management of neurogenic urinary incontinence but mainly in children with spina bifida $[1,2]$. Some studies included a small number of SCI patients [3, 4]. The outcome of the procedure was poor: efficacy was low, and there were high rates of complications including pseudoabscess and de novo urge incontinence [5]. There is evidence of regional and distant migration of polytetrafluoroethylene particles in animal and human studies
$[2,4]$. Therefore, bladder neck injection cannot be recommended for management of urinary incontinence in SCI patients.

\section{Bladder neck reconstruction}

To correct bladder neck incompetence reconstruction techniques have been developed including the Young-DeesLeadbetter procedure, the Kropp procedure, the Pippi Salle procedure, and bladder neck closure. Most studies relate to children with spina bifida. Evidence in SCI patients is insufficient for establishing specific recommendations. In the series of Nakamura et al., [6] one female SCI patient with severe intrinsic urethral sphincter deficiency achieved complete dryness in the long run. In the total group difficulty of catheterizing through the urethra (25\%), and urinary calculi (8\%) were complicating factors.

\section{Bladder neck closure}

Bladder neck closure is an irreversible procedure requiring urine drainage by a suprapubic outlet. It can be indicated in cases of intractable urinary incontinence, urethral erosion due to long-term indwelling catheters, scarring from previous trans-urethral procedures, and urethra-cutaneous fistula. The recommended technique is that described by Reid et al., [7] in which a transvesical resection of the bladder neck and prostatic urethra is performed. Then, the defect is closed with three overlapping purse-string sutures of 3-0 polyglycolic acid with watertight suture of the bladder mucosa on top.

Complete continence has been described in $40 \%$ [8]. Complications after bladder neck closure are not uncommon including leakage via stoma, urethral fistula, stomal complications, superficial wound infection [7-11],

\section{Peri-urethral autologous sling and synthetic tapes}

SCI may cause stress urinary incontinence (SUI) by intrinsic sphincter deficiency (ISD), and, sometimes, a coexisting urethral hypermobility. Autologous fascial sling surgery can restore urethral support and compress the urethra.

Mid-urethral synthetic tape operations, such as the transvaginal tape (TVT) or transobturator tape (TOT) procedures, have generally been reported in case series, and no study had a population entirely of individuals with SCI. A recent systematic review gave results for synthetic tape in only 20 individuals and for autologous fascial sling in 177 [12].

TOT placement gave $>80 \%$ full continence in smaller series [13] but other studies gave much lower positive outcome [14]. Complications (erosion and fistula) are rare, but 
in patients who void by straining a postoperative change, intermittent catheterization may be needed.

TVT placement gave similar positive results in the few patients published in the literature [15]. Comparison between synthetic tapes and autologous sling remains difficult due to small series.

There are few data on the use of alloplastic slings in male SCI patients. In small series, acceptable good results with TOT and less with the retropubic adjustable system have been described [16].

\section{Artificial urinary sphincter (AUS)}

The artificial urinary sphincter is considered the gold standard for ISD in male patients with NBD in several guidelines [17], but little data is available in SCI and results differ substantially between studies, mostly in meningomyelocele patients [18]. In general, better results are given when the cuff is placed around the bladder neck with less risk of erosion, more easy performance of cystoscopic procedures and clean intermittent catheterization (CIC), and minimal pressure through the perineal area when sitting in a wheelchair. Complications and reoperation rates are high $[12,19]$. Definitive AUS removal has been reported in up to $40 \%$ [19], possibly related to the younger age of the patients, more fragile tissues and comorbidities.

The question remains if placement of an AUS should be done simultaneously with procedures aimed at lowering detrusor pressure. Very acceptable results in favor of the simultaneous approach have been described by expert centers [17].

Robotically assisted bladder neck AUS implantation has been introduced [20]. Other novel modifications are bladder neck placement of a tissue expander port (instead of a pump) under the abdominal wall [21] and an AUS with conditional occlusion for SUI [22]. There is no data in SCI yet. Quality of life results are encouraging.

\section{Sphincterotomy and urethral stents}

If treatment with CIC and antimuscarinics is not successful, or side effects such as intolerable sphincterotomy or urethral stent insertion can lead to low-pressure bladder emptying by reflex contractions. Such a procedure should be limited to men who are able to wear a condom catheter. In women, or in obese men with a hidden penis, uncontrollable incontinence may occur. These procedures are invasive and especially sphincterotomy irreversible, while the patient has no possibility to first try out what the result would be [23]. Sphincterotomy has been shown to be an efficient technique for the resolution of autonomic dysreflexia (AD), hydronephrosis, and recurrent urinary tract infection (UTI), and for decreasing detrusor pressures, postvoid residual (PVR) urine, vesicoureteral reflux and hydronephrosis [24, 25]. The reoperation rate differs widely in the literature due to different techniques and outcome criteria. Complications described are hemorrhage requiring blood transfusion, and complete or partial loss of erection. The procedure of choice with less complications is the 12 o'clock sphincterotomy [26]. The use of the contact neodymium-doped yttrium aluminum garnet (Nd:YAG) laser in this setting gives similar results [27]. The technique is now less frequently used.

Though the first results with urethral stent as an alternative to conventional endoscopic sphincterotomy seemed promising [28], long-term results were not as satisfactory. Common complications are device migration, bladder neck obstruction, intravesical stone formation, encrustation, granulation tissue ingrowth, increase in $\mathrm{AD}$ and obstruction [29]. A temporary stent can be used in patients planning fertility treatment or in patients where the consequences of eventual sphincterotomy need to be better explored.

\section{Urinary diversion}

Where conservative treatment fails to control detrusor leak point pressure (DLPP), bladder compliance, and detrusor overactivity, more invasive surgical options need to be considered.

In general, continent methods of urinary reconstruction are preferred to incontinent techniques. However, between 1998 and 2005 (Nationwide Inpatient Sample (NIS) database, USA), 1132 patients with SCI underwent an augmentation, while 1909 underwent ileal conduit urinary diversion, showing that still a mere $0.08 \%$ of SCI patients got an incontinent procedure [30, 31].

The indications for incontinent diversion include compromised renal function (serum creatinine $>150-200 \mu \mathrm{mol} / \mathrm{L}$ ), hepatic and intestinal dysfunction, a deficient intrinsic sphincter, impaired cognitive ability, inability or unwillingness to perform CIC, lack of a reliable caregiver, patient preference, failure of continent diversion, requirement for bladder neck closure, intractable lower urinary tract obstruction and urethro-cutaneous fistula [31].

The ileal conduit is the most commonly used form of incontinent urinary diversion in NBD. The development of upper tract deterioration seems to increase with increasing length of follow-up. Pyocystis may develop and secondary cystectomy may become necessary. Stoma problems requiring intervention have been described in 5\%. Quality of life is generally improved [32].

A Cochrane review showed (with the limited data available) that there was no evidence to suggest that bladder reconstruction was better than conduit diversion. Likewise, 
there was no reported difference between ileal and colonic conduits with respect to pyelonephritis and uretero-intestinal anastomotic strictures. Similar results were seen with continent versus conduit diversion and, importantly, there was no difference in the rate of upper tract deterioration, unrelated to the type of anastomosis performed (refluxing vs. antirefluxing) [33].

Laparoscopic and robotic techniques are promising but their clinical value needs to be better determined. The decision to perform cystectomy at the time of diversion needs to be made, taking into account the potential for negative effects on the sexual function. Cystectomy can be done by bi-valving the bladder in a sagittal plane, amputating the lateral pedicles, and removing the urothelium on the residual trigone. The prostate may be left in situ [34].

Ileo-vesicostomy involves anastomosis of the rostral end of an isolated segment of ileum to the bladder, while the caudal end is put to the abdominal wall as an incontinent stoma. Laparoscopy and robot-assisted laparoscopy have been successfully performed in small series [35]. Current surgical concepts include a wide-mouthed vesical anastomosis, avoidance of limb redundancy, an adequate fascial window and a stoma conducive to appliance fit [36]. Where ISD exists prior to surgery, this needs to be managed by urethral or bladder neck closure, a pubo-vaginal sling, or AUS implantation. Advantages over ileal conduit urinary diversion include avoidance of uretero-intestinal stricture, no risk of pyocystis if the bladder is retained, no disturbance of the native upper tract anti-reflux mechanism and maintenance of sexual function. Contemporary case series give good results, both stomal stenosis and stone disease are uncommon, and excellent protection of the upper tract function. Caution should be taken in patients with morbid obesity [37]. Quality of life evaluation in this setting needs to be better studied. One has to take into account that the data in the literature are scant and many would not today recommend the technique as the bladder hardly never empties and there is risk of stones, infection and back pressure on the kidneys. These facts are not reported well.

Cutaneous vesicostomy is a procedure rarely performed in adults. The bladder dome is opened broadly and sutured directly to the skin as an incontinent stoma. Vesicostomy is not an effective management method in adults with SCI.

Cutaneous ureterostomy involves directly anastomosing the ureters to the skin as a non-continent form of diversion. Stenosis rates are high, as such, this procedure is rarely used today other than as a temporary measure prior to definitive therapy.

\section{Augmentation cystoplasty (AC)}

Indications for $\mathrm{AC}$ are detrusor overactivity with high intravesical pressure and/or low bladder compliance, both of which may lead to the development or progression of upper urinary tract deterioration and/or intractable urinary incontinence resistant to drug treatment and injection of botulinum neurotoxin type A in the detrusor. An inability to perform intermittent catheterization, inflammatory bowel diseases and to a certain point renal insufficiency are contraindications An upper limit of serum creatinine of approximately $2.0 \mathrm{mg} / \mathrm{dL}(176.8 \mu \mathrm{mol} / \mathrm{L})$ and a lower limit of creatinine clearance of approximately $50 \mathrm{~mL} / \mathrm{min}$ have been proposed, although the definitive cutoff values remain to be determined [38]. The bladder is usually incised like a clam shell, and enlarged by the implant of a detubularized intestinal segment in its wall aimed at creating a low-pressure-, high-capacity reservoir [39]. In the cases of a severely fibrotic bladder, some surgeons will perform a supra- or subtrigonal cystectomy making a longer bowel segment necessary to augment the bladder. Although AC is usually performed as open surgery, several reports of laparoscopic or robot-assisted laparoscopic AC, have recently been published in SCI cases [40]. Laparoscopic AC is a safe and technically feasible procedure, with a urodynamic outcome that is comparable to that reported for open AC. Complications are minor bowel disturbances, bladder stones, and perforation of the augmented bladder. The benefits of robot-assisted laparoscopic AC include decreased incisional pain, potentially decreased length of hospital stay, improved cosmetics, and decreased bowel and wound complications. These benefits need to be weighed against the steep learning curve, increased cost, and extended operation time of the robotic procedure.

Different technical points in the various surgical techniques described have been studied. Extraperitoneal AC provides an equally effective method of $\mathrm{AC}$ as the transperitoneal technique with earlier postoperative recovery [41].

There are advantages and disadvantages to each of the intestinal segments (ileum, sigmoid, ileocolic, stomach) used for A, but to discuss them in detail is beyond the scope of this manuscript.

Whether patients with vesicoureteral reflux should have ureteric reimplantation during the $\mathrm{AC}$ procedure remains uncertain. Follow-up after AC has shown the disappearance or significant improvement in reflux, meaning that the addition of ureteral reimplantation in AC is probably an unnecessary step for improving symptomatic UTI and preserving renal function [42].

The efficacy of ileum AC and colon AC has been documented in only few SCI patients as part of larger series with neurogenic bladder dysfunction. Urodynamic parameters significantly improved and nearly all achieved an adequate bladder capacity. Continence was achieved in the majority, symptomatic UTI was reduced and AD improved or disappeared in most patients. Many studies combined AC with concomitant bladder outlet procedures. But AC performed as the sole technique gave a high percentage of continence 
and significant improvement in quality of life which may be arguments in favor of a stepwise approach [42].

Early postoperative complications include anastomotic leakage, prolonged paralytic ileus, symptomatic UTI, and wound infection. Late complications can include bowel obstruction, bowel dysfunction causing diarrhea or loose stool, bladder and renal stone, symptomatic UTI, bowel-tobladder anastomotic stenosis, mucus secretion, metabolic acidosis, AUS infection, perforation and malignant neoplasms. Vitamin B 12 deficiency requiring supplementation was not reported in the literature. There is some risk for development of metabolic acidosis, requiring bicarbonate intake. Stone prevention with periodic bladder irrigation has not been studied in SCI patients. Perforation of the augmented bladder needs to be excluded when patients present with abdominal or general symptoms occur.

Malignant neoplasms (most frequently adenocarcinoma) have an estimated incidence from 0 to 272.3 per 100,000 patients/year and are diagnosed most frequently 10 years after surgery [43]. They are often found at an advanced stage which results in a poor prognosis. Annual cystoscopy surveillance is now the only validated tool available but gathering regular clinical data (UTI, pain, hematuria), associated with surveillance imaging and followed by selective cystoscopy may be sufficient [44].

The cost of AC has been compared to that of BoNTA but different evaluations give contradicting results based on the different approaches for comparison used [45].

Auto-augmentation has not been extensively studied in SCI patients. The best outcome could be expected in patients with good bladder capacity but poor bladder compliance. In this procedure, detrusor myotomy or myectomy is done, resulting in diverticulum-like expansion of the urothelial mucosa. In small series, maximum cystometric capacity improved, and maximum detrusor pressure and bladder compliance were normalized. Intra-operative mucosal perforation is the main surgical difficulty [46]. The long-term efficacy of auto-augmentation is not so good, with additional procedures required in $50 \%$ of patients.

In an experimental technique small intestinal submucosa (SIS) has been implanted into the bladder after a clam-shell cystostomy in a very limited number of SCI patients. Favorable results were described but more data in a higher number of patients are needed [47].

\section{Continent catheterizable channel indications}

In case of inability to perform ISC, when access to the urethra is practically impossible, or severe urethral pathology is present, creation of an abdominal drainage with suprapubic catheter or preferably with a continent catheterizable channel (CCC) should be considered. When indicated this is combined with augmentation cystoplasty, bladder neck closure, with or without AC. An appropriate stoma site should be carefully selected and will, in most cases, be located in the umbilicus, which is cosmetically acceptable and facilitates the finding of the opening for catheterization.

The tube is constructed using the appendix (Mitrofanoff procedure) [48], a retubularized short or double intestinal segment (Yang-Monti method, or Casale) [49, 50], or a similar method using the efferent limb of a Kock pouch or Indiana pouch [51].

There is a paucity of data in SCI patients.

\section{Sacral anterior root stimulation, posterior sacral root rhizotomy (SARS)}

The combination of sacral anterior root stimulation and posterior sacral root rhizotomy, also referred to as the "Brindley procedure", has been used for the restoration of bladder function after suprasacral SCI [52].

The implantable device (Finetech-Brindley SARS, Finetech Medical Limited, UK; Vocare ${ }^{\circledR}$ Bladder System, USA) works by stimulating motor pathways of sacral roots (S2-S4), which produces an effective contraction that can empty the bladder. The stimulator can also be used to support defecation and to restore penile erection. The stimulator is composed of an implanted part that consists of electrodes, cables, and a receiver that is placed subcutaneously, and an external part with a battery that gives wirelessly a transcutaneous current to the receivers, with a stimulation in the relevant nerves. In addition to the implant, the Brindley procedure also entails a sacral deafferentation, or dorsal sacral root rhizotomy, required to abolish NDO.

Three different surgical techniques have been used: intradural, extradural, and the "Barcelona", a combination of the other two.

Over the last 40 years, more than 2000 patients have undergone the Brindley procedure worldwide. In general, the acceptance of the Brindley implant by the patients is positive. Because of the posterior rhizotomy, a majority of the subjects in all studies were dry at follow-up. Other benefits of the Brindley procedure are reduction of UTI, reduction of AD episodes, and increase of bladder capacity. Quality of life improved significantly.

Despite these favorable results, the Brindley procedure has not been widely adopted, due to a lack of knowledge of the procedure, fear of surgery, uncertainty of the effects of the surgery and, above all, the irreversibility of the posterior rhizotomy and effects on reflex defecation and sexual function. These can be partly controlled by the stimulator itself.

Prospective case-control or cohort studies are warranted in the future. 


\section{Sacral neuromodulation}

The exact neural mechanisms responsible for the effects of electrical neuromodulation on the lower urinary tract are unknown. A dominant theory is that electrical stimulation of the somatic afferent fibers modulates voiding and continence reflex pathways in the CNS through a mechanism of gate control [53], resulting in the restoration of the balance between bladder inhibitory and excitatory control systems. Sacral neuromodulation might alter cortical sensory areas of the brain [54]. Neuromodulation may correct DSD and the inability to void by mediating an alteration of the afferent signals delivered to the spinal cord that affect activity and basal tone of the pelvic floor [55].

Neuromodulation through sacral nerve stimulation has been shown to improve maximum cystometric bladder capacity, resolve neurogenic detrusor overactivity [56] and improve baseline voiding parameters in cases with urinary retention with subsequent reduction frequency of catheterizations [57]. In the long run, nerve fatigue may limit the effectiveness of SNM requiring reimplantation on another nerve [58]. More data in a larger number of patients are needed to make a proper advice of the use of the technique feasible.

A study aimed to investigate the effect of bilateral SNM in SCI patients during the "spinal shock" phase on final bladder function after resolution of spinal shock. Further results in more patients are needed to come to conclusive responses if such action can improve the LUT function in the long run [58].

Adverse events are minor but lead migration, lack of efficacy, and dysfunction after trauma have been described [59].

Pudendal nerve stimulation may be an alternative technique to SNM [60] as may neuromodulation of the posterior tibial nerve, however, data for their use are sparse.

\section{Conclusion}

There are different surgical techniques that can be used to correct or improve neurogenic lower urinary tract dysfunction and its symptoms in those patients with spinal cord injury where conservative management is unsuccessful.

The choice of technique depends on the type of dysfunction found in bladder, bladder neck and urethra, or their combination. Furthermore, drugs and catheterization may still be required after surgery to provide a balanced and urodynamically safe lower urinary tract. In this context, surgical treatment should be seen as just one, albeit important part of the comprehensive global management of these patients.
Author contributions JJW, BB, AB, FB, DCD, ECK, MD, OI, TM, $\mathrm{EO}, \mathrm{KP}, \mathrm{BPF}, \mathrm{CR}$, and NS: paper development, data management, data analysis, manuscript writing and editing.

\section{Compliance with ethical standards}

Conflict of interest All authors declare that they have no conflict of interest.

Informed consent Not applicable as there are no study participants.

Research involving human participants and/or animals Not applicable as there are no human participants and/or animals.

\section{References}

1. Dyer L, Franco I, Firlit CF, Reda EF, Levitt SB, Palmer LS (2007) Endoscopic injection of bulking agents in children with incontinence: dextranomer/hyaluronic acid copolymer versus polytetrafluoroethylene. J Urol 178:1628-1631

2. Cole EE, Adams MC, Brock JW 3rd, Pope JC 4th (2003) Outcome of continence procedures in the pediatric patient: a single institutional experience. J Urol 170:560-563

3. Bennett JK, Green BG, Foote JE, Gray M (1995) Collagen injections for intrinsic sphincter deficiency in the neuropathic urethra. Paraplegia 33:697-700

4. Pérez LM, Smith EA, Parrott TS, Broecker BH, Massad CA, Wodard JR (1996) Submucosal bladder neck injection of bovine dermal collagen for stress urinary incontinence in the pediatric population. J Urol 156:633-636

5. Lightner DJ, Fox J, Klingele C (2010) Cystoscopic injections of dextranomer hyaluronic acid into proximal urethra for urethral incompetence: efficacy and adverse outcomes. Urology 75:1310-1314

6. Nakamura S, Hyuga T, Kawai S, Nakai H (2015) Long-term outcome of the Pippi Salle procedure for intractable urinary incontinence in patients with severe intrinsic urethral sphincter deficiency. J Urol 194:1402-1406

7. Reid R, Schneider K, Fruchtman B (1978) Closure of the bladder neck in patients undergoing continent vesicostomy for urinary incontinence. J Urol 120:40-42

8. Nguyen HT, Baskin LS (2003) The outcome of bladder neck closure in children with severe urinary incontinence. J Urol 169:1114-1116

9. Bergman J, Lerman SE, Kristo B, Chen A, Boechat MI, Churchill BM (2006) Outcomes of bladder neck closure for intractable urinary incontinence in patients with neurogenic bladders. J Pediatr Urol 2:528-533

10. Leslie B, Lorenzo AJ, Moore K, Farhat WA, Bägli DJ, Pippi Salle JL (2011) Long-term followup and time to event outcome analysis of continent catheterizable channels. J Urol 185:2298-2302

11. Welk BK, Afshar K, Rapoport D, MacNeily AE (2008) Complications of the catheterizable channel following continent urinary diversion: their nature and timing. J Urol 180:1856-1860

12. Farag F, Koens M, Sievert KD, De Ridder D, Feitz W, Heesakkers J (2016) Surgical treatment of neurogenic stress urinary incontinence: a systematic review of quality assessment and surgical outcomes. Neurourol Urodyn 35:21-25

13. Losco GS, Burki JR, Omar YA, Shah PJ, Hamid R (2015) Longterm outcome of transobturator tape (TOT) for treatment of stress urinary incontinence in females with neuropathic bladders. Spinal Cord 53:544-546 
14. Pannek J, Bartel P, Gocking K (2012) Clinical usefulness of the transobturator sub-urethral tape in the treatment of stress urinary incontinence in female patients with spinal cord lesion. J Spinal Cord Med 35:102-106

15. Lombardi G, Musco S, Celso M, Ierardi A, Nelli F, Del Corso F, Del Popolo G (2013) A retrospective study on female urological surgeries over the 10 years following spinal cord lesion. Spinal Cord 51:688-693

16. Pannek J, Wollner J (2017) Treatment of stress urinary incontinence in men with spinal cord injury: minimally invasive $=$ minimally effective? Spinal Cord 55:739-742

17. Chartier Kastler E, Genevois S, Gamé X, Denys P, Richard F, Leriche A, Saramon JP, Ruffion A (2011) Treatment of neurogenic male urinary incontinence related to intrinsic sphincter insufficiency with an artificial urinary sphincter: a French retrospective multicentre study. BJU Int 107:426-432

18. Singh G, Thomas DG (1996) Artificial urinary sphincter in patients with neurogenic bladder dysfunction. Br J Urol 77:252-255

19. Murphy S, Rea D, O'Mahony J, McDermott TE, Thornhill J, Butler M, Grainger R (2003) A comparison of the functional durability of the AMS 800 artificial urinary sphincter between cases with and without an underlying neurogenic etiology. Ir J Med Sci 172:136-138

20. Yates DR, Phé V, Rouprêt M, Vaessen C, Parra J, Mozer P, Chartier-Kastler E (2013) Robot-assisted laparoscopic artificial urinary sphincter insertion in men with neurogenic stress urinary incontinence. BJU Int 111:1175-1179

21. Bersch U, Göcking K, Pannek J (2009) The artificial urinary sphincter in patients with spinal cord lesion: description of a modified technique and clinical results. Eur Urol 55:687-693

22. Knight SL, Susser J, Greenwell T, Mundy T, Craggs MD (2006) A new artificial urinary sphincter with conditional occlusion for stress urinary incontinence: preliminary clinical results. Eur Urol 50:574-580

23. Noll F, Sauerwein D, Stöhrer M (1995) Transurethral sphincterotomy in quadriplegic patients: long-term-follow-up. Neurourol Urodyn 14:351-358

24. Perkash I (2007) Transurethral sphincterotomy provides significant relief in autonomic dysreflexia in spinal cord injured male patients: long-term followup results. J Urol 177:1026-1029

25. Vainrib M, Reyblat P, Ginsberg DA (2014) Long-term efficacy of repeat incisions of bladder neck/external sphincter in patients with spinal cord injury. Urology 84:940-945

26. Madersbacher H (1975) The twelve o'clock sphincterotomy: technique, indications, results. Paraplegia 13:261-267

27. Perkash I (1994) Laser sphincterotomy and ablation of the prostate using a sapphire chisel contact tip firing neodymium: YAG laser. J Urol 152:2020-2024

28. Rivas DA, Chancellor MB, Bagley D (1994) Prospective comparison of external sphincter prosthesis placement and external sphincterotomy in men with spinal cord injury. J Endourol 8:89-93

29. van der Merwe A, Baalbergen E, Shrosbree R, Smit S, Heyns C (2012) Outcome of dual flange metallic urethral stents in the treatment of neuropathic bladder dysfunction after spinal cord injury. J Endourol 26:1210-1215

30. Peterson AC, Curtis LH, Shea AM, Borawski KM, Schulman KA, Scales CD Jr (2012) Urinary diversion in patients with spinal cord injury in the United States. Urology 80:1247-1251

31. Cameron AP, Rodriguez GM, Gursky A, He C, Clemens JQ, Stoffel JT (2015) The severity of bowel dysfunction in patients with neurogenic bladder. J Urol 194:1336-1341

32. Guillotreau J, Castel-Lacanal E, Roumiguié M, Bordier B, Doumerc N, De Boissezon X, Malavaud B, Marque P, Rischmann P, Gamé X (2011) Prospective study of the impact on quality of life of cystectomy with ileal conduit urinary diversion for neurogenic bladder dysfunction. Neurourol Urodyn 30:1503-1506

33. Cody JD, Nabi G, Dublin N, McClinton S, Neal DE, Pickard R, Yong SM (2012) Urinary diversion and bladder reconstruction/ replacement using intestinal segments for intractable incontinence or following cystectomy. Cochrane Database Syst Rev. https://doi. org/10.1002/14651858.CD003306

34. Rowley MW, Clemens JQ, Latini JM, Cameron AP (2011) Simple cystectomy: outcomes of a new operative technique. Urology 78:942-945

35. Vanni AJ, Stoffel JT (2011) Ileovesicostomy for the neurogenic bladder patient: outcome and cost comparison of open and robotic assisted techniques. Urology 77:1375-1380

36. Tan HJ, Stoffel J, Daignault S, McGuire EJ, Latini JM (2008) Ileovesicostomy for adults with neurogenic bladders: complications and potential risk factors for adverse outcomes. Neurourol Urodyn 27:238-243

37. Schwartz SL, Kenneky MJ, McGuire EJ, Faerber GJ (1994) Incontinent ileovesicostomy urinary diversion in the treatment of lower urinary tract dysfunction. J Urol 152:99-102

38. Mast P, Hoebeke P, Wyndaele JJ, Oosterlinck W, Everaert K (1995) Experience with augmentation cystoplasty. A review. Paraplegia 33:560-564

39. Reyblar P, Ginsberg DA (2008) Augmentaion cystoplasty: what are the indications? Curr Urol Rep 9:452-458

40. El-Feel A, Abdel-Hakim MA, Abouel-Fettouh H, Abdel-Hakim AM (2009) Laparoscopic augmentation ileocystoplasty: results and outcome. Eur Urol 55:721-727

41. Reyblat P, Chan KG, Josephson DY et al (2009) Comparison of extraperitoneal and intraperitoneal augmentation enterocystoplasty for neurogenic bladder in spinal cord injury patients. World J Urol 27:63-68

42. Zhang P, Yang Y, Wu ZJ et al (2015) Should simultaneous ureteral reimplantation be performed during sigmoid bladder augmentation to reduce vesicoureteral reflux in neurogenic bladder cases? Int Urol Nephrol 47:759-764

43. Biardeau X, Chartier-Kastler E, Rouprêt M, Phé V (2016) Risk of malignancy after augmentation cystoplasty: a systematic review. Neurourol Urodyn 35:675-682

44. Lee HE, Bae J, Oh JK, Oh SJ (2013) Is concomitant bladder neck reconstruction necessary in neurogenic incontinent patients who undergo augmentation cystoplasty? Korean J Urol 54:42-47

45. Watanabe JH, Campbell JD, Ravelo A et al (2010) Cost analysis of interventions for antimuscarinic refractory patients with overactive bladder. Urology 76:835-840

46. Stöhrer M, Kramer A, Goepel M et al (1995) Bladder autoaugmentation - an alternative for enterocystoplasty: preliminary results. Neurourol Urodyn 14:11-23

47. Zhang F, Liao L (2014) Tissue engineered cystoplasty augmentation for treatment of neurogenic bladder using small intestinal submucosa: an exploratory study. J Urol 192:544-550

48. Cendron M, Gearhart JP (1991) The Mitrofanoff principle. Technique and application in continent urinary diversion. Urol Clin $\mathrm{N}$ Am 18:615-621

49. Monti PR, de Carvalho JR, Arap S (2000) The Monti procedure: applications and complications. Urology 55:616-621

50. Casale AJ (1999) A long continent ileovesicostomy using a single piece of bowel. J Urol 162:1743-1745

51. Rowland RG, Mitchell ME, Bihrle R et al (1987) Indiana continent urinary reservoir. J Urol 137(6):1136-1139

52. Brindley GS (1994) The first 500 patients with sacral anterior root stimulator implants: general description. Paraplegia 32:795-805

53. Leng WW, Chancellor MB (2005) How sacral nerve stimulation neuromodulation works. Urol Clin N Am 32:11-18

54. Blok BF, Groen J, Bosch JL, Veltman DJ, Lammertsma AA (2006) Different brain effects during chronic and acute sacral 
neuromodulation in urge incontinent patients with implanted neurostimulators. BJU Int 98:1238-1243

55. Schmidt RA, Jonas U, Oleson KA, Janknegt RA, Hassouna MM, Siegel SW, van Kerrebroeck PE (1996) Sacral nerve stimulation for treatment of refractory urinary urge incontinence. Sacral Nerve Stimulation Study Group. J Urol 162:352-357

56. Chartier-Kastler EJ, Denys P, Chancellor MB, Haertig A, Bussel B, Richard F (2001) Urodynamic monitoring during percutaneous sacral nerve neurostimulation in patients with neurogenic detrusor hyperreflexia. Neurol Urodyn 20:61-71

57. Lombardi G, Del Popolo G (2009) Clinical outcome of sacral neuromodulation in incomplete spinal cord injured patients suffering from neurogenic lower urinary tract symptoms. Spinal Cord 47:486-491

58. Sievert K, Amend B, Gakis G, Badke A, Kaps HP, Stenzl A (2010) Early sacral neuromodulation prevents urinary incontinence after complete spinal cord injury. Ann Neuro 67:74-84

59. White WM, Mobley JD 3rd, Doggweiler R, Dobmeyer-Dittrich C, Klein FA (2009) Incidence and predictors of complications with sacral neuromodulation. Urology 7:731-735

60. Opisso E, Borau A, Rodríguez A, Hansen J, Rijkhoff NJ (2008) Patient controlled versus automatic stimulation of pudendal nerve afferents to treat neurogenic detrusor overactivity. J Urol 180:1403-1408 